ORIGINAL ARTICLE

\title{
Differences in kinematic parameters between male and female hammer throw finalists of the World Championship in Daegu in 2011
}

Ratko Pavlović $\mathrm{ABCDE}$

University of East Sarajevo, Bosnia and Herzegovina

Authors' Contribution: A - Study design; B - Data collection; C - Statistical analysis; D - Manuscript Preparation; E - Funds Collection

\begin{tabular}{|c|c|}
\hline \multicolumn{2}{|l|}{ Abstract } \\
\hline Purpose: & $\begin{array}{l}\text { Hammer throwing is the most complex athletic throwing discipline with rotational trajectory and } \\
\text { strong effect of several different forces that try to disable the projected throwing trajectory. Kinematic } \\
\text { parameters are an important segment in the analysis of athletic disciplines, including hammer throw. } \\
\text { They are an indicator of influence and often a difference between competitors of different or the same } \\
\text { rank of the competition. The aim of the study was to determine the spatial and temporal differences of } \\
\text { kinematic parameters between male and female elite hammers. }\end{array}$ \\
\hline Material: & $\begin{array}{l}\text { The study was conducted on sample of } 16 \text { Daegu World Championship finalists in } 2011 \text {, to analyse } \\
\text { differences in kinematic parameters between male and female throwers hammer. To obtain the required } \\
\text { results, t-test for small independent samples was applied. }\end{array}$ \\
\hline Results: & $\begin{array}{l}\text { The data obtained in the study were given as Mean and Standard deviation. Statistically significant } \\
\text { differences between male and female finalists were confirmed in the rate of ejection }(t=3.684 ; p<0.004) \\
\text { and the speed of the fourth turn ( } t=4.396 ; p<0.002 \text { ). The male finalists achieved an average ejection } \\
\text { velocity of } 27.91 \mathrm{~m} / \mathrm{s} \text { and the female finalists } 27.17 \mathrm{~m} / \mathrm{s} \text {, with an average turn speed of } 4.67 \mathrm{~m} / \mathrm{s} \text { (male) and } \\
4.03 \mathrm{~m} / \mathrm{s} \text { (female). }\end{array}$ \\
\hline Conclusions: & $\begin{array}{l}\text { The research has shown that significant differences were made between male and female finalists in } \\
\text { Daegu in } 2011 \text {. The causes of differences can be found in length of training, different training process, } \\
\text { technical mastery, competitor experience, morphological profile, motor and anatomical structures, } \\
\text { movement technique and biomechanical parameters, which were not taken into the research. }\end{array}$ \\
\hline Keyn & World Championship, kinematic parameters, hammer throw, differences. \\
\hline
\end{tabular}

\section{Introduction}

Throws are movements that shift certain weights over some distance, affect muscle mass, increase the strength of the entire body, and especially the muscles of the arms, shoulder girdle and the entire body. They also, to certain extent, develop agility and speed, and they are part of phylogenetic form of movement that, over time, with continuous training process, translates into forms of various ontogenetic forms, the so-called athletic throws. One such ontogenetic form is hammer throw. The main objective is to throw the hammer as far as possible in accordance with the rules of the competition. From historical distance, hammer throwing is related to the Celtic tradition, where originally hammer with wooden handle was thrown at competitions. This hammer throw has continued to this day in popular contests among the Irish and the Scots. Then rotate around their vertical shafts like today's. By 1878, the hammer was thrown quite primitively, firmly standing from the line drawn and since then from a circle $2,135 \mathrm{~m}$ in diameter. It is mostly thrown from the standing point, with no turns, and later with one or two turns in circular swing and throwing out. The turn is made on the heel, and after ejection step forward is usual, while the length of the shot is measured from the place where the ejector stops after the ejection. After the dominance of American competitors (Totell,

(c) Ratko Pavlović, 2020

doi:10.15561/26649837.2020.0506
Callaghan, Quinn), in the thirties began the dominance of European throwers. Everyone is trying to find the best movement technique for successful shot. Particularly standing out were German (Hein, Blask) who introduced the so-called German version of hammer throw. This variant is modified by Comstok, Nemet, and later Russian Krivonosov, Bonadrchuk, Spiridonov introducing new so-called Russian version of hammer throw with the aim of increasing the radius of the spin in hammer system [1].

Generally, in uneducated subjects, the opinion is that because of their constitution, is less effective in displaying motor skills, which is wrong. They have high level of development and manifestation of motor skills during motor movement [2]. In addition to motor skills, an important place is occupied by the body composition of the thrower. According to Ugarković [3], male and female throwers are dominated by muscle mass (53$56 \%$ ), bone mass $(18-22 \%)$ and fat $(15-19 \%)$. Based on these parameters, it can be concluded that in the thrower the primary is mesomorphic with the endomorphic and ectomorphic component, according to which the hammer throwers correspond to the meso-endomorphic somatotype (3.75-5.50-2.25). Pavlović, Radinović, \& Janković [4], Pavlović, Raković, Radić, Simeonov, \& Piršl [5] consider that throwers have greater amount of muscle mass compared to other athletes and are closest to the mesomorphic type according to Sheldon's classification. They have lower body height $(190 \mathrm{~cm})$ and 
larger body weight $(113 \mathrm{~kg})$ which is positively correlated with performance. From motor point of view, all athletic throws, including hammer throws, are initiated by the explosive activation of agonist muscles, followed by period of relaxation, and end with deceleration period due to the action of the antagonist muscles or the passive stretching of connective tissue with an integrated involvement of particular motor skills (static, repetitive and explosive power, flexibility, balance, anaerobic potential). Hammer throw is characterized by the maximum speed of movement being achieved through rotations around the vertical axis in the sagittal plane to the moment of ejection, which requires a kinaesthetic feel, high degree of motor skills, above all speed power, coordination of movement, excellent orientation during the execution of turns and sense of rhythm. Top throwers perform three or four turns (the official world record of Soviet thrower Yuriy Sedikh is $86.74 \mathrm{~m}$ at the $19^{\text {th }}$ European Championship in Stuttgart and was achieved with three turns) [1]. It was thought that four turns were performed by lower and more technically trained (faster) throwers and three turns by more powerful throwers. However, today, both variants are common with all throwers, with four turns and even five turns being more common. At turns, the thrower and the hammer do not only rotate around the vertical axis, but they also move in the direction of the throw as the speed of rotation increases $[6,7]$. During the turn, large centrifugal force develops, transferring to the thrower, trying to knock them down. In order to counteract the fall, the thrower tilts to the opposite side of the hammer (the so-called counterweight). The size of the pitch of the thrower's body to the opposite side of the hammer depends on the thrower's own weight, physical fitness and technical skill. The greater the weight of the thrower, as well as his strength, the lower the incline. Maintaining the vertical position of the body while turning is almost impossible, but it should be pursued, because if the axis of rotation is left unchanged, the hammer rotation lever increases, and ultimately the flight distance of the device increases too [8]. Identical to other throwing disciplines, hammer flight distance depends on the initial flight velocity, the angle of ejection, and the height of the ejection point [9], of which speed has proven to be the most important [10, 11]. According to Maroński [12], an increase in ejection velocity dramatically improves the range of any projectile throw, while Bowerman, Freeman, \& Vern Gambetta [13] believe that an increase in velocity by $5 \%$ can increase the result by 7 meters while changing the angle by $5 \%$ alters the length for only $60 \mathrm{~cm}$. From a biomechanical point of view, it is primary for hammer throwers to communicate as much speed (through turns) as possible within a short time interval within the limits of the support surface [1], despite the effect of gravitational force.

In addition to the influence of the primary factors in the resultant success, gravitational, centrifugal and Coriolos forces act as well [14]. Using computer modelling for typical ejection heights and optimum hammer ejection angles, the impact of Earth rotation on the hammer throw and collision distance with air currents, air pressure, temperature, altitude, and earth slope, presented practical maps for technique correction to achieve results at different latitudes and/or different release directions can be corrected by a formula that incorporates the effect of earth's rotation. The hammer movement system in space includes phenomena that affect the functional flow of the ultimate speed of the device [15]. The spatial trajectory of the hammer head and the corresponding anthropometric points are evaluated from the standpoint of single turns, double and single support and ejection phases, studying the tangential and acceleration components as well as the forces acting on the device. Positive factors have been found to cause an increase in the speed of the hammer head, including several factors (Strong foot action, with the feet constantly rotating and never held in a static double-pivot position; two turns of length; Rotation of the torso in front of the pelvis, with displacement of the centre of the shoulder line connecting the right hip joint; rotation of the shoulder axis in front of the hammer axis; vertical lifting of the hip joints in the direction of vertical movement of the hammer and hammer head; obtuse angle between the shoulder axis and the hammer, with the highest possible position of the device in the range of 1.60 to 2.00 meters at the beginning of the ejection phase. Dapena, \& Mc Donald [16], using three-dimensional cinematography, recorded the angular momentum of elite throwers, their trajectory, and vector accelerations.

They proved that the trajectory of the angular momentum vector, the incline of the body and the height of the hammer plane with respect to the mass system of the thrower are interrelated. Some throwers hold the hammer handle high (the body tilts back in all turns), while other throwers hold the hammer handle low (the body tilts forward in the initial turns), so in later turns the hammer handle rises as the body tilts back. They also suggested some theories to explain why athletes who lean forward in the first turn, in the back of the throw, lean back. Dapena, Gutiérrez-Dávila, Soto, \& Rojas-Ruiz [17] analyse the resultant hammer throw performance as consequence of neutralizing air flow resistance, assuming the centre of mass of the hammer coincides with the centre of the ball. To calculate kinematic parameters in hammer ejection, they use three-dimensional throwing data for male and female competitors using a mathematical model simulation. The distance provided for the vacuum and centre of gravity of the hammer ball is approximately $4.30 \mathrm{~m}$ longer than the official throwing distance for men and $8.80 \mathrm{~m}$ longer for women. Predictions of use of the right centre of mass of the hammer reduced the deviations for men $(2.39 \pm 2.58 \mathrm{~m})$ and women $(5.28 \pm 2.88 \mathrm{~m})$, while the predictions of the action of air resistance and the true centre of mass of the hammer further reduced the deviations for the men $(-0.46 \pm 2.63 \mathrm{~m})$ and women's $(1.16 \pm 2.31 \mathrm{~m})$. Half of the loss of distance produced by the air resistance was due to the action of forces on the ball and the rest due to the forces exerted on the cable and the hammer handle. It is an indisputable fact that increasing the force of the hammer wire when turning is crucial for throwing distance. Brice, Ness, Rosemond, 
Lyons, \& Davis [18] analysed the top five hammer throws and calculated the force acting on the hammer head from Newton's second law of motion, and then compared it with the force measured by stress apparatus. The results showed that the qualitative time dependence of the two forces was essentially the same, while the quantitative average difference between the measured and calculated forces over the five throws was $76 \mathrm{~N}$, corresponding to difference of $3.8 \%$ for the hammer wire force of $2000 \mathrm{~N}$. Also, Brice, Ness, \& Rosemond [19] on sample of male and female throwers, analysed the relationship of force and linear velocity of the hammer wire when throwing, and identified the influence of the magnitude and direction of the force on the fluctuation of the hammer speed. The results showed strong correlation between the decrease in linear velocity and the hammer wire force (normalized to its weight). A strong correlation was also found between the angle at which the hammer force lags behind in its rotation radius to its maximum (when the tangential force is most negative) and the magnitude of the hammer speed decrease. All top hammer throwers are known to have good anatomical and physiological muscle structure that allows for top-notch technique execution.

The study by Terzis, Spengos, Kavouras, Manta, $\&$ Georgiadis [20] confirmed that the efficiency of hammer throwing is highly correlated with the athlete's lean body mass and higher bone mineral density. They have also proven that the percentage of type IIA muscle fibre surface is over $66 \%$ with more than 8000 fibres in the muscle structure. According to Ojanen, Rauhala, \& Häkkinen [21] maximum power, explosive power, muscle thickness, and power characteristics decline with aging, and with throwers who have been actively practicing power and throwing for decades. However, with top athletes, the maximum strength, muscle mass as well as the explosive force of the upper and lower extremities appear to be at significantly higher levels than those recorded for men of the same age. A group of Brazilian authors [9] analysed differences in kinematic parameters (angle, velocity, ejection height, velocity curve as a function of time) between Brazilian and international throwers, starting with the 3D trajectory of the hammer head. The results showed the resultant inferiority of Brazilian throwers with respect to international throwers, in the ejection velocity (average $24.59 \mathrm{~m} / \mathrm{s}$ male and $23.59 \mathrm{~m} / \mathrm{s}$ female), international (average $29.60 \mathrm{~m} / \mathrm{s}$ male and $28.89 \mathrm{~m} / \mathrm{s}$ female). The velocity curves as function of the time of the international throwers compared to the Brazilian sample show that accelerations are higher and deceleration is smaller during the turn. The success in hammer throw depends on training, years of experience, training volume [22]. A survey of American throwers examined the parameters that contributed most to distance in hammer throwing. Multiple regression analysis for the personal hammer throw record provided significant model $(\mathrm{r}=0.811, \mathrm{p} \leq 0.001)$ with predictors (number of throws per year, number of hammer throws, back squat and type of hammer technique). The model explained more than $65 \%$ of the discrepancy in hammer technique among respondents, thus providing quantitative information for coaches and athletes involved in this throwing discipline. Also different anthropometric differences between the genders affect the technique and the end result of hammer throw. Konz, \& Hunter [23] on a sample of 16 male and female throwers of the 2003 World Athletics Finals and 13 male and female throwers from the USA in 2003 conduct research to determine differences in the technique of throwing between the genders. The results revealed that athlete mass, athlete height; ejection rate, some temporal components, and centripetal force were different between the two genders. Panoutsakopoulos, Vujkov, \& Obradović [24] on a sample of male and female competitors investigated the relationship between hammer throw duration and distance of three and four turns, and examined the relationship between hammer throw duration and hammer throw performance by two-line Pearson correlations. The results showed that there was a high and strong correlation between throwing duration and throwing distance for 4-turn throwers $(\mathrm{r}=-83 ; \mathrm{p}<0.01)$ and 3 turn throwers $(\mathrm{r}=-.96 ; \mathrm{p}<0.01)$. Most throwers during the technique of execution, spent more time in single-support than in double-support. It was concluded that the greater the throwing distance, the shorter the total turn duration, i.e. in the inverse relationship.

The main goal of this research was defined on the basis of the insight and review of the previous research and based on the previous research results. The aim of the current research is to analyse and determine statistically significant differences in kinematic parameters between male and female hammer throwers in the 2011 Daegu World Athletics Championship finals.

\section{Material and Methods}

\section{Participants}

The sample included 16 top Hammer throwers (8 male and 8 female) who competed in the 2011 World Championship finals in Daegu, average result male $79,06 \mathrm{~m}$ and female $73,80 \mathrm{~m}$.

\section{Procedure}

All data of kinematic parameters are taken from the Scientific Research Project Biomechanical Analyses at the 13 IAAF World Championship, Daeegu, 2011. Variables were identified for estimating of biomechanical parameters in Hammer throwers (Table 1).

1. Starting velocity $(\mathrm{m} / \mathrm{s})$

2. Velocity of release $(\mathrm{m} / \mathrm{s})$

3. Angle of Release $\left(^{\circ}\right)$

4. Increase of Velocity $(\mathrm{m} / \mathrm{s})$ :

a) Turn $1(\mathrm{~m} / \mathrm{s})$;

b) Turn $2(\mathrm{~m} / \mathrm{s})$;

c) Turn $3(\mathrm{~m} / \mathrm{s})$,

d) Turn $4(\mathrm{~m} / \mathrm{s})$

Statistical Analysis

The data obtained in the study were given as mean and standard deviation. Independent T-test for small samples was used, and the significance level was accepted as $\mathrm{p}<0.001$. Statistica 10.0 program was used in the statistical analysis of the study. 
Table 1. The Kinematic Parameters of Male and Female Finalists WCh, Daegu 2011. [37]

\begin{tabular}{|c|c|c|c|c|c|c|c|c|}
\hline \multirow[b]{2}{*}{ Male } & \multirow[b]{2}{*}{$\begin{array}{l}\text { Distance } \\
\text { (m) }\end{array}$} & \multirow{2}{*}{$\begin{array}{l}\text { Starting } \\
\text { Velocity } \\
(\mathrm{m} / \mathrm{s})\end{array}$} & \multicolumn{4}{|c|}{ Increase Velocity $(\mathrm{m} / \mathrm{s})$} & \multirow[b]{2}{*}{$\begin{array}{l}\text { Velocity of } \\
\text { Release }(\mathrm{m} / \mathrm{s})\end{array}$} & \multirow[b]{2}{*}{$\begin{array}{l}\text { Angle of } \\
\text { Release }\left({ }^{\circ}\right)\end{array}$} \\
\hline & & & $\begin{array}{l}\text { Turn } 1 \\
(\mathrm{~m} / \mathrm{s})\end{array}$ & $\begin{array}{l}\text { Turn } 2 \\
(\mathrm{~m} / \mathrm{s})\end{array}$ & $\begin{array}{l}\text { Turn } 3 \\
(\mathrm{~m} / \mathrm{s})\end{array}$ & $\begin{array}{l}\text { Turn } 4 \\
(\mathrm{~m} / \mathrm{s})\end{array}$ & & \\
\hline Murofushi & 81.24 & 16.7 & 3.4 & 2.4 & 1.3 & 4.6 & 28.3 & 41.4 \\
\hline Pars & 81.18 & 18.1 & 2.9 & 1.6 & 1.0 & 4.6 & 28.2 & 44.1 \\
\hline Kozmus & 79.39 & 16.4 & 3.5 & 2.2 & 1.2 & 4.7 & 28.1 & 39.0 \\
\hline Esser & 79.12 & 16.3 & 3.8 & 1.9 & 0.9 & 5.0 & 27.9 & 41.1 \\
\hline Kryvitski & 78.53 & 16.1 & 3.5 & 2.3 & 1.4 & 4.7 & 27.9 & 39.1 \\
\hline Ikonnikov & 78.37 & 15.9 & 3.7 & 2.4 & 1.2 & 4.6 & 27.7 & 44.1 \\
\hline Ziolkowski & 77.64 & 16.4 & 3.3 & 2.3 & 1.3 & 4.5 & 27.8 & 38.9 \\
\hline Vizzoni & 77.04 & 17.6 & 2.6 & 2.3 & 5.0 & - & 27.4 & 43.9 \\
\hline Mean & 79.06 & 16.69 & 3.33 & 2.12 & 1.66 & 4.67 & 27.91 & 41.5 \\
\hline \multirow[b]{2}{*}{ Female } & \multirow[b]{2}{*}{$\begin{array}{l}\text { Distance } \\
\text { (m) }\end{array}$} & \multirow{2}{*}{$\begin{array}{l}\text { Starting } \\
\text { Velocity } \\
(\mathrm{m} / \mathrm{s})\end{array}$} & \multicolumn{4}{|c|}{ Increase Velocity (m/s) } & \multirow[b]{2}{*}{$\begin{array}{l}\text { Velocity of } \\
\text { Release }(\mathrm{m} / \mathrm{s})\end{array}$} & \multirow[b]{2}{*}{$\begin{array}{l}\text { Angle of } \\
\text { Release }\left({ }^{\circ}\right)\end{array}$} \\
\hline & & & $\begin{array}{l}\text { Turn } 1 \\
(\mathrm{~m} / \mathrm{s})\end{array}$ & $\begin{array}{l}\text { Turn } 2 \\
(\mathrm{~m} / \mathrm{s})\end{array}$ & $\begin{array}{l}\text { Turn } 3 \\
(\mathrm{~m} / \mathrm{s})\end{array}$ & $\begin{array}{l}\text { Turn } 4 \\
(\mathrm{~m} / \mathrm{s})\end{array}$ & & \\
\hline Lysenko & 77.13 & 16.4 & 4.5 & 2.3 & 0.8 & 3.8 & 27.8 & 43.1 \\
\hline Heidler & 76.06 & 18.4 & 2.6 & 1.7 & 1.0 & 3.9 & 27.6 & 40.1 \\
\hline Zhang & 75.03 & 17.9 & 3.1 & 1.9 & 1.1 & 3.4 & 27.4 & 42.6 \\
\hline Moreno & 74.48 & 14.4 & 4.0 & 2.9 & 1.6 & 4.4 & 27.3 & 41.1 \\
\hline Wlodarczyk & 73.56 & 15.5 & 3.7 & 2.6 & 1.3 & 4.4 & 27.4 & 36.6 \\
\hline Perie & 72.04 & 16.2 & 2.9 & 2.4 & 0.9 & 4.4 & 26.8 & 40.3 \\
\hline Klaas & 71.89 & 17.5 & 2.8 & 1.8 & 0.8 & 3.9 & 26.7 & 41.9 \\
\hline Marghieva & 70.27 & 13.6 & 5.2 & 2.5 & 1.1 & 4.0 & 25.8 & 43.1 \\
\hline Mean & 73.80 & 16.2 & 3.6 & 2.26 & 1.07 & 4.02 & 27.1 & 41.1 \\
\hline
\end{tabular}

\section{Results}

Inspection of Table 2 revealed quantitative differences in all measured kinematic parameters between male and female finalists. Men achieved higher average starting speed $(16.69 \mathrm{~m} / \mathrm{s})$, they were faster in third $(1.66 \mathrm{~m} / \mathrm{s})$ and fourth turn $(4.67 \mathrm{~m} / \mathrm{s})$, while women had $(3.60 \mathrm{~m} / \mathrm{s})$ in first and $(2.26 \mathrm{~m} / \mathrm{s})$ in second turn. Both samples exhibited fluctuations in velocity increment and almost identical ejection angle. (Table 2). Statistically significant differences were observed only in the parameters of ejection rates $(\mathrm{T}=3.684 ; \mathrm{p}<0.004)$ and speed in the fourth turn $(\mathrm{T}=4.396 ; \mathrm{p}<0.002)$ in favour of male competitors. The average ejection velocity of the male finalists at Daegu is $(27.91 \mathrm{~m} / \mathrm{s})$, which is $0.73 \mathrm{~m} / \mathrm{s}$ faster than the female finalists, while the angle of ejection is minimal (Table 1,2). The highest ejection velocity was achieved by the first-ranked Murofushi $(28.3 \mathrm{~m} / \mathrm{s})$, with an ejection angle of $41.4^{\circ}$ and starting velocity of $16.7 \mathrm{~m} / \mathrm{s}$. The individual starting speed of the male finalists ranged from $15.9 \mathrm{~m} / \mathrm{s}$ (Ikonnikov) to $18.1 \mathrm{~m} / \mathrm{s}$ (Pars) and the ejection speed from $27.4 \mathrm{~m} / \mathrm{s}$ (Vizzoni) to $28.3 \mathrm{~m} / \mathrm{s}$ (first place Murofushi). The average initial velocity of ejaculation (starting velocity) of males was $16.69 \mathrm{~m} / \mathrm{s}$ and at ejection it was $27.91 \mathrm{~m} / \mathrm{s}$ (almost $28 \mathrm{~m} / \mathrm{s}$ ). It follows that during four turns the speed increased by $11.22 \mathrm{~m} / \mathrm{s}$ with considerable oscillations in the speed of turn (3.33-2.12-1.66-4.67m/s). The hammer was thrown with an average ejection angle of $41.5^{\circ}$, ranging from minimum of $38.9^{\circ}$ (Ziolkowski) to maximum of $43.9^{\circ}$ (Vizzoni). (Table 1; Figure 1, 3). It can be concluded that the speed increased with the number of turns, which is the goal of every hammer thrower, to maximize the speed at the moment of ejection. In addition to the statistical difference in ejection rate between finalists, the difference was made in the speed of execution of the last fourth turn, where the male finalists were faster by $64 \mathrm{~ms}$ (Table 1, 2; Figure 3 ).

When we talk about female throwers, Lysenko threw the hammer with the highest starting speed $(27.8 \mathrm{~m} / \mathrm{s})$ and slightly higher recoil angle of $43^{\circ}$ at starting speed of $16.4 \mathrm{~m} / \mathrm{s}$. Female throwers achieved lower starting speed than male finalists, ranging from $13.6 \mathrm{~m} / \mathrm{s}$ (Marghieva) to $18.4 \mathrm{~m} / \mathrm{s}$ (Heidler) with tendency to grow in the last turn. Also, women were inferior to the individual results of the ejection velocity from $26.4 \mathrm{~m} / \mathrm{s}$ (Marghieva) to $27.8 \mathrm{~m} / \mathrm{s}$ (Lysenko) and the average initial velocity of $10.9 \mathrm{~m} / \mathrm{s}$ (Table 2, Figures 2 and 3). The average initial velocity of ejaculation of females is $16.20 \mathrm{~m} / \mathrm{s}$ and at ejection is $27.10 \mathrm{~m} / \mathrm{s}$. Over the course of four turns, this speed increased by $10.90 \mathrm{~m} / \mathrm{s}$ with considerable oscillations in the speed of rotation (3.6-2.26-1.07-4.02m/ $\mathrm{s})$. The average ejection angle is $41.1^{\circ}$, with maximum of $43.1^{\circ}$ (Maghieva, Lysenko) and minimum of $36.6^{\circ}$ (Wlodarczyk). Marghi was last placed, with the lowest starting speed $(13.6 \mathrm{~m} / \mathrm{s})$ and the breaking speed $(25.8 \mathrm{~m} / \mathrm{s})$, unlike the male finalists, where the first-ranked Murofushi had the lower speed than the two competitors $(16.7 \mathrm{~m} / \mathrm{s})$, 
Table 2. The differences in kinematic parameters between male and female Hammer throwers

\begin{tabular}{|c|c|c|c|c|c|c|}
\hline \multicolumn{2}{|c|}{ The kinematic parameters } & Gender & Mean \pm St.Dev. & t-value & $\begin{array}{l}\text { p-value } \\
\text { (2-sided) }\end{array}$ & $95 \% \mathrm{Cl}$ \\
\hline \multirow{2}{*}{\multicolumn{2}{|c|}{ Starting Velocity $(\mathrm{m} / \mathrm{s})$}} & Male & $16.69 \pm 0.776$ & \multirow{2}{*}{0.687} & \multirow{2}{*}{0.508} & \multirow{2}{*}{-0.955 to 1.855} \\
\hline & & Female & $16.24 \pm 1.688$ & & & \\
\hline \multirow{2}{*}{$\frac{\tilde{n}}{\xi}$} & \multirow{2}{*}{ Turn 1 (m/s) } & Male & $3.34 \pm 0.403$ & \multirow{2}{*}{-0.739} & \multirow{2}{*}{0.477} & \multirow{2}{*}{$-1,024$ to 0,499} \\
\hline & & Female & $3.60 \pm 0.920$ & & & \\
\hline 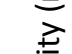 & \multirow{2}{*}{ Turn $2(\mathrm{~m} / \mathrm{s})$} & Male & $2.17 \pm 0.282$ & \multirow{2}{*}{-0.486} & \multirow{2}{*}{0.635} & \multirow{2}{*}{$-0,473$ to 0,298} \\
\hline 흠 & & Female & $2.26 \pm 0.424$ & & & \\
\hline $\bar{\Phi}$ & \multirow{2}{*}{ Turn $3(\mathrm{~m} / \mathrm{s})$} & Male & $1.66 \pm 1.359$ & \multirow{2}{*}{1.200} & \multirow{2}{*}{0.267} & \multirow{2}{*}{$-0,463$ to 1,638} \\
\hline \multirow{3}{*}{ 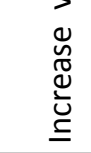 } & & Female & $1.08 \pm 0.271$ & & & \\
\hline & \multirow{2}{*}{ Turn 4 (m/s) } & Male & $4.67 \pm 0.160$ & \multirow{2}{*}{4.396} & \multirow{2}{*}{$0.002 * *$} & \multirow{2}{*}{0,329 to 0,964} \\
\hline & & Female & $4.03 \pm 0.358$ & & & \\
\hline \multirow{2}{*}{\multicolumn{2}{|c|}{ Release of velocity (m/s) }} & Male & $27.91 \pm 0.290$ & \multirow{2}{*}{3.684} & \multirow{2}{*}{$0.004 * *$} & \multirow{2}{*}{0.308 to 1.167} \\
\hline & & Female & $27.17 \pm 0.486$ & & & \\
\hline \multirow{2}{*}{\multicolumn{2}{|c|}{ Angle of release $\left(^{\circ}\right)$}} & Male & $41.45 \pm 2.336$ & \multirow{2}{*}{0.311} & \multirow{2}{*}{0.760} & \multirow{2}{*}{-2.065 to 2.765} \\
\hline & & Female & $41.10 \pm 2.164$ & & & \\
\hline
\end{tabular}

NOTE: Mean (average value), standard deviation (St.Dev), coefficient t-test (t-value), Sig.level $\left({ }^{* *} p<0,001\right), \mathrm{Cl} \%=$ confidence interval

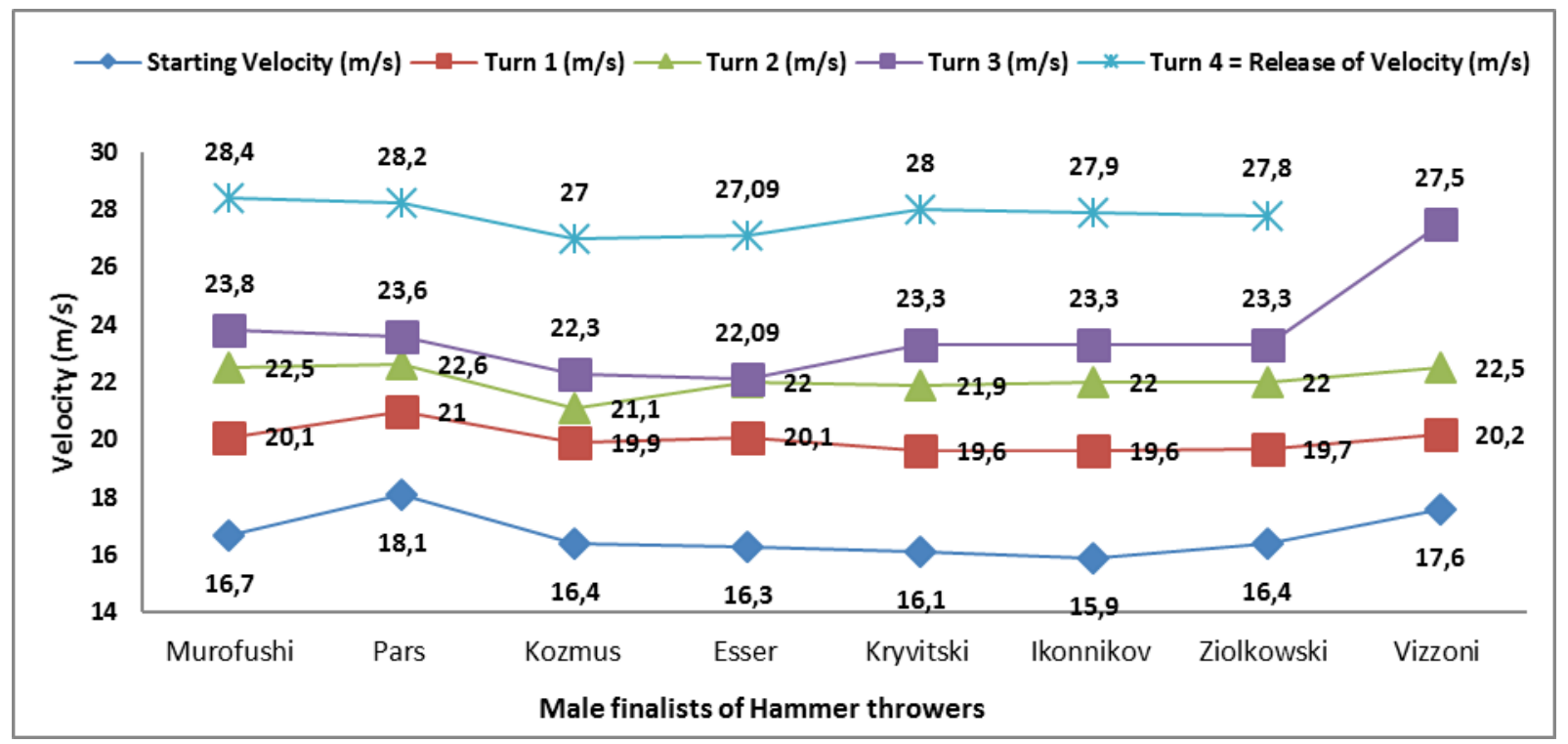

Figure 1. Distribution of male results in kinematics parameters

but still managed to achieve the highest ejection rate (Table 1; Figures 2, 3). It can be concluded that he had good maximum speed gain through four turns and good movement technique $(11.6 \mathrm{~m} / \mathrm{s})$.

\section{Discussion}

The main objective of the study was to determine the differences in kinematic parameters between male and female finalists in the 2011 World Athletic Championship in Daegu. Results were obtained confirming the significant differences between the genders, which is the main result of the research. The question arises as to the cause of such results?

First of all, hammer throw competition is considered to be one of the most complex throwing competitions in terms of technique and difficulty encountered in training [25]. It is complex movement that requires the expression of different forces and the harmony of the movements of the competitors [2], and depends on the type of training, years of experience and volume of training [22]. Coordination is the most complex technique of the throwing discipline, so high results at the international level require continuous improvement. The technique of movement depends on achieving the maximum speed of the hammer through the turns up to the moment of device ejection [26, 27], the angle and height of the ejection [13]. The technique integrates rotational motion in which there is reciprocal action of the thrower-hammer system, which always rotates about the shaft passing between the centre of gravity of the thrower body and the hammer, as well 


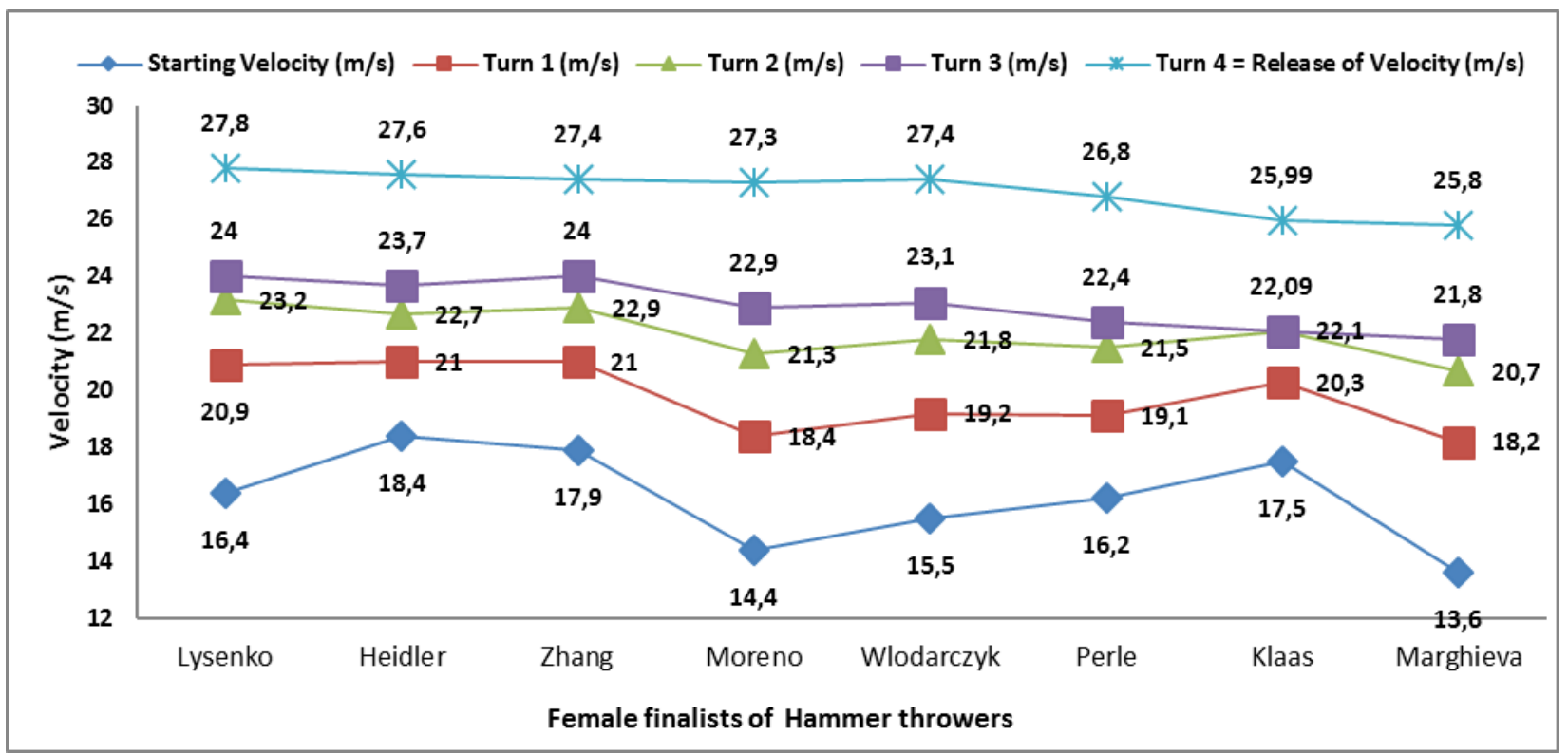

Figure 2. Distribution of female results in kinematics parameters

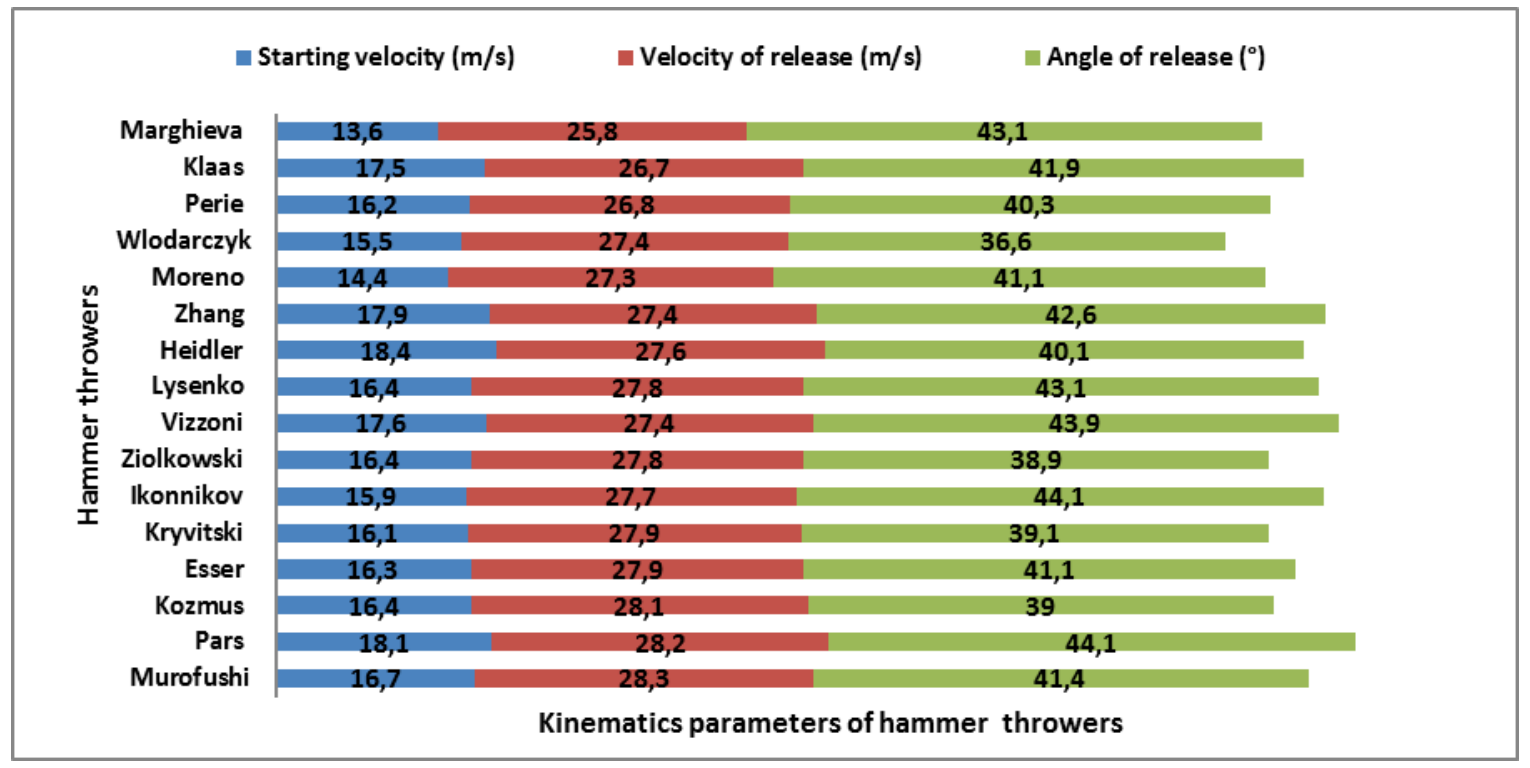

Figure 3. Distribution of individual results competitors

as through the support and the surface. Each movement begins with the initial swinging of the thrower, creating the conditions for entry into the first turn, allowing the thrower to reach the necessary rhythm and good concentration, which is important for the further stages of the technique. The number of turns performed depends primarily on the training of the thrower, his anatomical and physiological structures, and motor skills [20]. This is followed by sweeps, where the competitor rotates with the hammer around the vertical axis. During the hammer waving, the initial turning speed $(12-16 \mathrm{~m} / \mathrm{s})$ is announced and the common centre of gravity of the system (throwerhammer) must be within the limits of the support surface $[1,7]$. The results of the current survey are in accordance with the above, where the average starting speed ranges from $16.2 \mathrm{~m} / \mathrm{s}$ (women) to $16.7 \mathrm{~m} / \mathrm{s}$ (men).

In the hammer override phase, the movement of the launcher is directed to achieve the highest speed of rotation $[23,24]$, where the launcher and the hammer form unique system that performs complex rotational motion by rotating the launcher around its vertical axis, while moving in a sagittal plane, from the last part of the circle towards the front maximum speed, i.e. achieve maximum speed and act on the device in the ejection phase [11]. If the coordination of the thrower and thus the turns, is better, the speed of each subsequent turn is higher and the fluctuation of the speed is recorded. As its velocity increases, the centrifugal force $(2900-4000 \mathrm{~N})$ increases, affecting the balance of the competitor, seeking to dislodge it from the imaginary path which counteracts the action of strong centrifugal force by tilting the body in backward compensatory movements of the leg joints, bending the knees lowering the centre of gravity of the body $[1,6,7$, $8]$. In order to make the swing as large as possible, the 
thrower makes certain twists with soft movements in the joints of the spine and hips. When the hammer is moving away from the body, the thrower moves the pelvic part of the body opposite to the device, accelerating the hammer, performing a second pre-swing, creating the preconditions for the start of the second or third swing $[1,6,9]$. During the rotation, the hammer speed gradually increases and reaches velocity close to $28 \mathrm{~m} / \mathrm{s}$ at the moment of ejection [23] while the ideal ejection angle is $40^{\circ}$, although some top throwers throw at an angle of $38^{\circ}$ to $40^{\circ}$ [13]. However, some authors believe that the angle depends on the height of the athlete and ranges from 42 to $44^{\circ}[7,8,28]$, which is contrary to the results of this research. The average ejection angle of finalists in Daegu is $41^{\circ}$ with individual higher or lower competitor values, which is consistent with the allegations [13]. Štuhec, Vertič, \& Čoh [11] found the largest differences in ejection velocity $(2.6 \mathrm{~m} / \mathrm{s})$ in the analysis between Y. Sedikh and P. Kozmus, while the ejection angle and height were almost identical, which is similar to the results of this study (Table 2, Figures 1-3).

Hammer throw is characterized by complex spatial and temporal structure, with the aim of achieving maximum movement speed through two or three movements and moving in 3, 4 or 5 turns, moving linearly through the centre of the throwing circle. Such movement further complicates changes in the spatial orientation of planes that define the trajectory of the hammer movement in each turn [29]. Each thrower in one turn goes through two clearly differentiated periods: double-backing (entry of the thrower into the turn) and single-backing (exit of the thrower from the turn). It is common opinion that the hammer acceleration is achieved in the doublebacking contact phase (the hammer pull force is directed downwards and forwards to build the stability of the thrower and to achieve greater speed of movement) rather than the one-backing (the hammer pull force is very strong and is counteracted by the deflection of the hull which is the opposite of the hammer position). Both phases are of very short duration $(0.20-0.26 \mathrm{~s})$, which decrease as one goes from the first to the fourth turn. Also, at turns, the distance between the feet decreases and speed increases, from the initial $16 \mathrm{~m} / \mathrm{s}$ to over $28 \mathrm{~m} / \mathrm{s}$ at the moment of ejection, as confirmed by the results of this study (Table 1, Figure 3). Based on this theory, some coaches [30, 31] have tried to extend the double-backed support phase and have largely contributed to the excellent results of Soviet throwers in recent decades. The theory is proved by the marked fluctuation in the speed of the hammer in each double-backing support and noticeable decrease in the single-backing support.

However, Dapena $[16,28]$ questioned this theory, so he proved that it is possible to increase the speed even in a single-backing support. He believes that the impact of the thrower on the hammer speed cannot be negative, even when the thrower pulls the hammer towards the body, because it comes from the highest point of the trajectory. Exactly in our sample there is a fluctuation in speed through turns, where female finalists recorded slightly higher average speeds in the first and second turns
(Turn $1=3.6 \mathrm{~m} / \mathrm{s}$; Turn $2=2.26 \mathrm{~m} / \mathrm{s}$ ) than the male (Turn $1=3.33 \mathrm{~m} / \mathrm{s}$; Turn $2=2.12 \mathrm{~m} / \mathrm{s}$ ) and the third and fourth turns are dominated by male finalists (Turn $3=1.66 \mathrm{~m} / \mathrm{s}$; Turn $4=4.67 \mathrm{~m} / \mathrm{s}$ ), with this that there were statistically significant differences between the finalists in the fourth turn (Table 2). According to some authors $[1,7,8]$, the turning of the hammer begins when it is on the right side, below shoulder height. At the moment of passing over the arch of the left foot, the velocity of the hammer head is the highest and therefore the strongest centrifugal force, so the incline of the thrower is necessary for balance. When entering the first turn, the shoulders and hips are parallel, however, when exiting the turn and moving into the single-backing period, the hips move faster, overtaking the device, in order to achieve double double-backing period as quickly as possible. At the moment of the doublebacking period, the first turn is completed, generating high rotation moment [16]. At this stage, the thrower seeks to achieve the best anatomical-biomechanical parameters for the most efficient and favourable entry into the rotation through synchronous myometrial-plyometric muscle contractions across muscle kinetic chains. So, the hammer in the second turn must enter at higher speed than it was the entry into the first. The same principle is used to enter and exit the next three turns. This theory was confirmed in our study for both sub-samples, where from starting speed of about $17 \mathrm{~m} / \mathrm{s}$, the ejection velocity increased by $11 \mathrm{~m} / \mathrm{s}$ successively, from turn to turn, with maximum speed in the fourth turn at the ejection stage (Table 1 Figure 1-3), which is consistent with research [11]. For instance, according to Y. Sedikh, the speed of rotation is gradually increasing through the turns, so that at the moment of ejection this speed would be record even today $(30.7 \mathrm{~m} / \mathrm{s})$.

The timing of the top throwers' rotation depends on the duration of the number of turns and the speed increment, ranging from $1.64 \mathrm{sec}$. (with 3 turns) up to $2.16 \mathrm{sec}$. (with 4 turns). Murofushi performed 4 turns in $1.96 \mathrm{sec}$, Tikhon in $2.04 \mathrm{sec}$, when reaching a result over $80 \mathrm{~m}$, etc. The speed of ejection, if given the hammer at the right angle, is almost always a crucial factor of the end result $[1,6]$. When throwing hammers, the action on the long road is not a guarantee of good results, but it is necessary to communicate the maximum force in the shortest time interval [24]. The movement of the hammer is performed with two to three swings, which are then followed by three or four turns in which the thrower rotates in synchronization, the speed of the hammer being steadily increased until the moment of ejection [26]. In the technical embodiment, the circular motion of the hammer around the thrower, the gradual change in the inclination of the plane of movement of the hammer and the horizontal trajectory of the thrower-hammer system within the circle are observed. In the initial part of the throw, the hammer is in the horizontal question, at $37^{\circ}$ [8], but becomes steeper as speed increases and reaches incline of about $40^{\circ}$ during the last turn. The thrower keeps the hammer in its circular path and the centrifugal force, during the last turn, is transmitted through the wire 
to the centre of the ball. In twist, the wire acts equally and opposite to the force of the thrower's hand, which tends to pull it forward [1, 7, 16, 19].

Each gender has characteristics that contribute to success, as different anthropometric differences occur between the genders that cast the hammer and are likely to influence the optimal technique [23]. The most visually noticeable differences are in height and body mass, where male hammer throwers are typically taller and have more mass than their counterparts $[4,5]$. The male hammer is proportionally more massive than the female hammer and relative to the body mass, which results in different mastering of the inertia force and the centrifugal force. Therefore, female throwers have higher starting speed due to less body mass, shorter torso and less ball weight, as well as the centre of mass of rotation due to the difference in weight and mass distribution during turns and throws $[10,19,33]$. Women are more upright during throwing because of their lower body mass and lighter weight, so they require less effort than men when confronting inertia forces and centrifugal force [34-37].

Generally speaking, the results obtained in this study for the female sample are partly consistent with previous research regarding the ejection rate, starting speed, and turning speed of female throwers, especially the first and second turns.

\section{Conclusion}

The study was conducted on a sample of 16 finalists at the 2011 Daegu World Athletics Championships to determine differences in kinematic parameters between male and female hammer throwers. The results of the study confirmed differences in all defined parameters, but statistically significant differences of finalists were recorded only in the rate of ejection $(\mathrm{T}=3.684 ; \mathrm{p}<0.004)$ and the speed of the fourth turn $(T=4.396 ; p<0.002)$ in favour of men. Consequences of differences can be found in length of training, different training process, competitor experience, morphological profile, motor and anatomical structures, movement technique, biomechanical parameters not taken into the research. Future research could consider a larger number of kinematic parameters and larger number of subjects.

\section{Conflict of interests}

The author states that there is no conflict of interests.

\section{References}

1. Pavlović R. Athletics-textbook, Niš. Association of writers Branko Miljkovic; 2016.

2. Milanović D. Training theory. Zagreb: Faculty for Kinesiology; 1997.

3. Ugarković D. Biology of human development with the basics of sports medicine. Beograd: Faculty of Physical education and Sport; 1996.

4. Pavlović R, Radinović Z, Janković M. Morphological status of finalists in the throwing disciplines at the Beijing Olympics. Sportmont. 2012; 10 (34-36): 447-455.

5. Pavlović R, Raković A, Radić Z, Simeonov A, Piršl D. Morphological status of female athletes in throwing disciplines at the Olympic games in Beijing. Research in Physical Education, Sport and Health. 2013; 2 (2):113-119.

6. Idrizović K. Athetics I \& II-textbook. Library of Biomedical research. Podgorica; 2010.

7. Mihajlović I. Athletics-textbook. Novi Sad: Faculty of Physical education and Sport; 2010.

8. Stefanović Đ, Bošnjak G. Athletics-textbook, philosophicalscientific basics and application in practice. Banja Luka: Faculty of Physical education and Sport; 2011 (In Serbian)

9. Mercadante LA, Menezes RP, Martini TP, Trabanco JLA, Leite de Baros RM. 3D Kinematical Analysis of The Hammer Throw In Competitions, 2007. 25 International Symposium on Biomechanics in Sports, Ouro Preto - Brazil; 2007. P. 314-331.

10.Baronietz K, Barclay L, Gathercole D. Characteristics of top performances in the women's hammer throw: basics and technique of the world's best athletes. New Studies in Athletics. 1997; 12 (2-3): 101-109.

11. Štuhec S, Vertič R, Čoh M. 3D kinematic analysis of the hammer throw-Primož Kozmus. In monograph M.Čoh (ed.), Biomechanical diagnostic methods in athletic training (8591). Ljubljana: Faculty of Sport, Institute of Kinesiology; 2008.

12.Maroński R. Optimal distance from the implement to the axis of rotation in hammer and discus throws. $J$ Biomech. 1991; $24 \quad$ (11):999-1005. https://doi.org/10.1016/0021-9290(91)90017-H

13.Bowerman WJ, Freeman WH, Vern Gambetta TAC. Atleticshigh-perfomance training for track and field. Zagreb-Gopal; 1998.

14.Mizera F, Horváth G. Influence of environmental factors on shot put and hammer throw range. Journal of Biomechanics. 2002; 35 (6): 785-796. https://doi.org/10.1016/S0021-9290(02)00029-5

15.Susanka P, Stepanek J, Miskos G, Terauds J. Hammer-Athlete Relationship During The Hammer Throw, 4 International Symposium on Biomechanics in Sports, 1986; 1:194-200.

16.Dapena J, McDonald C. A three-dimensional analysis of angular momentum in the hammer throw. Medicine and Science in Sports \& Exercise. 1989;21(2): 206-220. https://doi.org/10.1249/00005768-198904000-00015

17.Dapena J, Gutiérrez-Dávila M, Soto V, RojasRuiz F. Prediction of distance in hammer throwing. Journal of Sport Sciences. 2003; 21 (1): 21-28. https://doi.org/10.1080/026404103100007092

18. Brice SM, Ness KF, Rosemond D, Lyons K, Davis M. Development and validation of a method to directly measure the cable force during the hammer throw. Sports Biomechanics. 2008; 7(2): 274-87. https://doi.org/10.1080/14763140701841902

19. Brice SM, Ness KF, Rosemond D. An analysis of the relationship between the linear hammer speed and the thrower applied forces during the hammer throw for male and female throwers. Journal Sports Biomechanics. 2011; 10(3): 174-184. https://doi.org/10.1080/14763141.2011.592210

20.Terzis G, Spengos K, Kavouras S, Manta P, Georgiadis G. Muscle fibre type composition and body composition in hammer throwers. J Sports Sci Med. 2010; 9(1):104-109.

21.Ojanen T, Rauhala T, Häkkinen K. Strength and power profiles of the lower and upper extremities in master throwers at different ages. J Strength Cond Res.2007; 21(1):216-222. https://doi.org/10.1519/00124278-200702000-00039

22.Judge L, Bellar D, Mc Atee G, Judge M. Predictors 
of Personal Best Performance in the Hammer Throw for U.S. Collegiate Throwers. International Journal of Performance Analysis in sport.2010; 10(1): 54-65. https://doi.org/10.1080/24748668.2010.11868501

23.Konz S, Hunter I. Technique Comparison of Male And Female Hammer Throwers. 33 International Conference of Biomechanics in Sports, Coaching and Sports Activities, 2015. P.1038-1041.

24.Panoutsakopoulos V, Vujkov N, Obradović B. The duration of the throw is correlated with the throwing distance in men's and women's hammer throwing with three and four turns. Modern Athlete and Coach, 2012;50 (1): 33-39.

25.Elalem Mohamed, SF. Evaluation of Hammer Throw Technique for Faculty of Physical Education Students using DartFish Technology. Journal of Applied Sports Science. 2016; 6 (2): 80-87. https://doi.org/10.21608/jass.2016.84559

26. Maheras A. Reassessing velocity generation in hammer throwing. New studies in Athletics. 2009; 24 (4): 71-80.

27.Shesterova L, Rozhkov V. Interrelation of the hammer swing technique with the technique of its previous rotation in highly skilled hammer throwers. Slobozhanskyi Herald of Science and Sport. 2018; 6 (68):13-16. https://doi.org/10.15391/snsv.2018-6.003

28.Dapena J. The Pattern of Hammer Speed During a Hammer Throw and Influence of Gravity on its Fluctuations. Journal of Biomechanics. 1984; 17(8): 553-559. https://doi.org/10.1016/0021-9290(84)90086-1
29.Gutiérrez-Davila M, Soto VM, Rojas-Ruiz FJ. A biomechanical analysis of the individual techniques of the hammer throw finalists in the Seville Athletics World Championship 1999. New studies in Athletics, 2002; 17 (2): 15-26.

30.Bondarchuk AP. The modern technique of hammer throwing. Athletics Austria. 1979; 2(3): 22-23.

31.Bondarchuk AP. Modern technique in hammer throw. Athletics Notebooks, 1987; 20: 65-72.

32.Knudson DV. Fundamentals of Biomechanics. New York: Kluwer Academic/Plenum Publishers; 2003. https://doi.org/10.1007/978-1-4757-5298-4

33.Hay JG. The Biomechanics of Sports Techniques. Upper Saddle River, New Jersey: Prentice Hall; 1993.

34.Ransdell LB, Wells CL. Sex Differences in Athletic Performance. Women in Sport and Physical Activity Journal, 1999;8:55-81. https://doi.org/10.1123/wspaj.8.1.55

35.Bartonietz K. Arremesso de martelo: problemas e perspectives. In: Zatsiorsky VM. Biomecânica no Esporte. Rio de Janeiro: Guanabara-Koogan; 2004. P. 358-380.

36.Dapena J.AKinematics Study of Center of Mass Motion in the HammerThrow.JounalofBiomechanics. 1986;19(2):147-158. https://doi.org/10.1016/0021-9290(86)90144-2

37.Isele R, Hoo LS, Nixdorf E. Biomechanical Analysis of the Hammer Throw Men and Women Final. Scientific Research Project Biomechanical Analyses at the 13 IAAF World Championship, Daeegu, 2011 Final Report Hammer Throw; 2011.

\section{Information about the author:}

Ratko Pavlović; http://orcid.org/0000-0002-4007-4595; pavlovicratko@yahoo.com; Faculty of Physical Education and Sport, University of East Sarajevo; East Sarajevo, Bosnia and Herzegovina.

Cite this article as:

Pavlović R. Differences in kinematic parameters between male and female hammer throw finalists of the World Championship in Daegu in 2011. Pedagogy of physical culture and sports, 2020;24(5):255-263. https://doi.org/10.15561/26649837.2020.0506

This is an Open Access article distributed under the terms of the Creative Commons Attribution License, which permits unrestricted use, distribution, and reproduction in any medium, provided the original work is properly cited (http://creativecommons.org/licenses/by/4.0/deed.en).

Received: 03.04.2020

Accepted: 15.05.2020; Published: 30.10 .2020 